\title{
Adaptive Synchronization of Complex Dynamical Networks with State Predictor
}

\author{
Yuntao Shi, ${ }^{1}$ Bo Liu, ${ }^{2}$ and Xiao Han ${ }^{2}$ \\ ${ }^{1}$ Key Laboratory of Beijing for Field-Bus Technology \& Automation, North China University of Technology, Beijing 100144, China \\ ${ }^{2}$ College of Science, North China University of Technology, Beijing 100144, China
}

Correspondence should be addressed to Bo Liu; boliu@ncut.edu.cn

Received 12 July 2013; Accepted 27 August 2013

Academic Editor: Michael Chen

Copyright (C) 2013 Yuntao Shi et al. This is an open access article distributed under the Creative Commons Attribution License, which permits unrestricted use, distribution, and reproduction in any medium, provided the original work is properly cited.

This paper addresses the adaptive synchronization of complex dynamical networks with nonlinear dynamics. Based on the Lyapunov method, it is shown that the network can synchronize to the synchronous state by introducing local adaptive strategy to the coupling strengths. Moreover, it is also proved that the convergence speed of complex dynamical networks can be increased via designing a state predictor. Finally, some numerical simulations are worked out to illustrate the analytical results.

\section{Introduction}

In recent years, the synchronization problem of complex networks is a hot topic in many areas, including mathematics, physics, biology, computer, and artificial intelligence [1-37]. A complex dynamical network is composed of a large number of nodes to be steered to arrive synchronization. However, it is so hard and impractical to control all nodes to synchronize in the large complex network. In order to solve this problem, the pinning control is introduced, which can decrease the number of controllers for synchronization of the complex networks. Pinning control scheme is an effective way to control dynamical networks to a desired state. The idea of the pinning control is to steer a small fraction nodes, and all nodes of the complex network can achieve control target via localized feedback of those nodes.

A lot of outstanding works about the synchronization problem of complex networks are presented in the recent references [1-37]. In [1], Wang and Chen used specifically and randomly pinning control strategies for scale-free chaotic dynamical networks. De Lellis et al. [19] considered the synchronization of complex networks through local adaptive coupling. The authors [20] proposed a decentralized adaptive pinning control scheme for synchronization of undirected networks using a local adaptive strategy to both coupling strengths and feedback gains. In [4], Yu et al. investigated the synchronization via pinning control on general complex networks. In $[5,6]$, second-order consensus problem for multiagent systems was investigated by using pinning control method.

The convergence speed of complex dynamical networks is a significant issue. Through limited communication, each agent can forecast the future states of its neighbors and itself; as well as the new control law can be constructed by the predicted states. With this strategy, the complex dynamical network can evolve more quickly to equilibrium. Motivated by it, this paper investigates the synchronization via designing a state predictor. It is proved that all nodes will asymptotically synchronize to the given homogeneous stationary state using the adaptive strategy to the coupling strengths designed, if the complex dynamical network is connected and at least one node is informed. Introducing the state predictor for formation algorithm in multiagent systems, the simulation results show that using the state predictor in multiagent systems can improve the speed of the system to complete the desired task.

The rest of this paper is designed as follows. Section 2 gives a model of the complex dynamical network. Some preliminaries are introduced to solve the adaptive synchronization. Section 3 provides the theoretical analysis of adaptive synchronization of the complex dynamical network. Furthermore, some more detailed analyses are presented in 
this section. Section 4 gives some simulations to illustrate our theoretical results. Conclusion is finally summarized in Section 5 .

\section{Preliminaries and Problem Statement}

Consider a complex dynamical network described by

$$
\begin{aligned}
\dot{x}_{i}(t)= & f\left(x_{i}(t)\right)-\sum_{j \in \mathcal{N}_{i}} a_{i j} c_{i j}(t)\left(x_{i}(t)-x_{j}(t)\right) \\
& +\gamma \sum_{j \in \mathcal{N}_{i}} a_{i j} c_{i j}(t)\left(\dot{x}_{i}^{p}(t)-\dot{x}_{j}^{p}(t)\right),
\end{aligned}
$$

where $x_{i}(t)=\left(x_{i 1}(t), x_{i 2}(t), \ldots, x_{i n}(t)\right)^{T} \in R^{n},(i=$ $1,2, \ldots, N)$ represents the state vector of the node $i$ at time $t$; $N_{i}$ is the neighbor set of node $i ; f(\cdot) \in R^{n}$ is continuously differentiable; $a_{i j}$ is the coupling weight between any two agents, where $a_{i j} \geq 0$ and $a_{i i}=0 ; c_{i j}(t)$ denotes the coupling strengths between nodes $i$ and node $j$; the weighted coupling configuration matrix of the system is defined as

$$
U=\left[u_{i j}\right]=\left[\begin{array}{cccc}
u_{11} & u_{12} & \cdots & u_{1 N} \\
u_{21} & u_{22} & \cdots & u_{2 N} \\
\vdots & \vdots & \ddots & \vdots \\
u_{N 1} & u_{N 2} & \cdots & u_{N N}
\end{array}\right] \in R^{N \times N}
$$

with $u_{i i}=a_{i i} c_{i i}=-\sum_{j=1, j \neq i}^{N} a_{i j} c_{i j}$ and $u_{i j}=a_{i j} c_{i j}$ for $i \neq j$. Design the state predictor for the control law as

$$
\dot{X}^{p}=-L X
$$

where $\dot{X}^{p}=\left(\dot{x}_{1}^{p}, \dot{x}_{2}^{p}, \ldots, \dot{x}_{N}^{p}\right)$ and $\gamma$ is the impact factor of the state predictor.

Under state predictor (3), network (1) can be rewritten as

$$
\begin{aligned}
\dot{x}_{i}(t)= & f\left(x_{i}(t)\right)-\sum_{j \in \mathcal{N}_{i}} a_{i j} c_{i j}(t)\left(x_{i}(t)-x_{j}(t)\right) \\
& -\gamma\left(\sum_{j \in \mathcal{N}_{i}} \sum_{k \in \mathcal{N}_{i}} a_{i j} a_{i k} c_{i j}(t) c_{i k}(t)\left(x_{i}-x_{k}\right)\right. \\
& \left.-\sum_{j \in \mathcal{N}_{i}} \sum_{p \in \mathcal{N}_{j}} a_{i j} a_{j p} c_{i j}(t) c_{j p}(t)\left(x_{j}-x_{p}\right)\right) .
\end{aligned}
$$

Definition 1. Network (4) is said to achieve synchronization if

$$
\lim _{t \rightarrow \infty}\left\|x_{i}(t)-\bar{x}(t)\right\|=0, \quad i=1, \ldots, N
$$

where the homogeneous state satisfies

$$
\dot{\bar{x}}(t)=f(\bar{x}(t), t)=0 .
$$

The adaptive control at node $i$ is designed as

$$
\begin{aligned}
\dot{c}_{i j}(t)= & \sum_{p \in \mathcal{N}_{j}} a_{i j} a_{j p} c_{j p}(t)\left[x_{i}(t)-\bar{x}(t)\right]^{T}\left[x_{i}(t)-\bar{x}(t)\right] \\
& +\sum_{k \in \mathcal{N}_{i}} a_{i j} a_{i k} c_{i k}(t)\left[x_{k}(t)-\bar{x}(t)\right]^{T}\left[x_{k}(t)-\bar{x}(t)\right],
\end{aligned}
$$

where $c_{i j}(0) \geq 0$.

In the following, some necessary assumptions and lemmas are stated.

Assumption 2. The coupling strengths of the network are bounded:

$$
\left\|c_{i j}(t)\right\| \leq c_{i j}
$$

Assumption 3 (see [5]). The vector field $f_{i}: R^{n} \rightarrow R^{n}(i=$ $1,2, \ldots, N)$ in network (4) satisfies the Lipschitz condition; there exists a positive constant $\rho>0$, such that

$$
\|f(x)-f(y)\| \leq \rho\|x-y\|
$$

Assumption 4. The weights satisfy the balance condition:

$$
\sum_{j \in N_{i}} a_{i j} c_{i j}(t)=\sum_{j \in N_{i}} a_{j i} c_{j i}(t), \quad \forall i
$$

Lemma 5 (see [29]). For any vectors $x, y \in R^{n}$ and positive definite matrix $G \in R^{n \times n}$, the following matrix inequality holds:

$$
2 x^{T} y \leq x^{T} G x+y^{T} G^{-1} y .
$$

Lemma 6 (see [6]). Supposing that $a$ and $b$ are vectors, then for any positive-definite matrix E, the following inequality holds:

$$
-2 a^{T} b \leq \inf _{E>0}\left\{a^{T} E a+b^{T} E^{-1} b\right\}
$$

Lemma 7 (see [38]). The matrix A of an undirected graph $G$ is irreducible if and only if the undirected graph is connected.

\section{Main Results}

In this section, we will give detailed analysis of the adaptive synchronization of the network with the state predictor. By using the Lyapunov function approach, adaptive synchronization conditions of such work are obtained.

Theorem 8. Considering network (4) with $N$ nodes steered by adaptive control (7), under Assumptions 2-4, then all nodes 
will asymptotically synchronize to the given homogeneous stationary state; that is,

$$
\lim _{t \rightarrow \infty}\left\|x_{i}(t)-\bar{x}(t)\right\|=0
$$

Proof. Let $\widetilde{x}_{i}(t) \triangleq x_{i}(t)-\bar{x}(t)$. Construct the following Lyapunov function:

$$
V(t)=V_{1}(t)+V_{2}(t)
$$

where

$$
\begin{gathered}
V_{1}(t)=\frac{1}{2} \sum_{i=1}^{N} \widetilde{x}_{i}^{T}(t) \tilde{x}_{i}(t), \\
V_{2}(t)=\sum_{i=1}^{N} \sum_{j \in N_{i}} \frac{\left[(1-m) c_{i j}(t)\right]^{2}}{2},
\end{gathered}
$$

where $m>0$ is sufficiently large.

By the definition of the matrix $U$, it is to see that $U$ is symmetric and irreducible. By Lemmas $5-7$, differentiating $V_{1}(t)$, we can have

$$
\begin{aligned}
& \dot{V}_{1}(t)=\sum_{i=1}^{N} \tilde{x}_{i}^{T}(t)\left[f\left(x_{i}(t)\right)-f(\bar{x}(t))\right] \\
& -\sum_{i=1}^{N} \widetilde{x}_{i}^{T}(t) \sum_{j \in \mathcal{N}_{i}} a_{i j} c_{i j}(t)\left(\tilde{x}_{i}(t)-\tilde{x}_{j}(t)\right) \\
& -\gamma \widetilde{x}_{i}^{T}(t) \sum_{j \in \mathcal{N}_{i}} \sum_{k \in \mathcal{N}_{i}} a_{i j} c_{i j}(t) a_{i k} c_{i k}(t)\left(\widetilde{x}_{i}(t)-\widetilde{x}_{k}(t)\right) \\
& +\gamma \widetilde{x}_{i}^{T}(t) \sum_{j \in \mathcal{N}_{i}} \sum_{p \in \mathcal{N}_{j}} a_{i j} c_{i j}(t) a_{j p} c_{j p}(t)\left(\widetilde{x}_{j}(t)-\widetilde{x}_{p}(t)\right) \\
& \leq \rho \sum_{i=1}^{N} \tilde{x}_{i}^{T}(t) \tilde{x}_{i}(t)-\sum_{i=1}^{N} \sum_{j \in \mathcal{N}_{i}} a_{i j} c_{i j}(t) \tilde{x}_{i}^{T}(t) \tilde{x}_{i}(t) \\
& +\sum_{i=1}^{N} \sum_{j \in \mathscr{N}_{i}} a_{i j} c_{i j}(t) \tilde{x}_{i}^{T}(t) \tilde{x}_{j}(t) \\
& -\gamma \sum_{i=1}^{N} \sum_{j \in \mathscr{N}_{i}} \sum_{k \in \mathcal{N}_{i}} a_{i j} c_{i j}(t) a_{i k} c_{i k}(t) \tilde{x}_{i}^{T}(t) \tilde{x}_{i}(t) \\
& +\gamma \sum_{i=1}^{N} \sum_{j \in \mathcal{N}_{i}} \sum_{k \in \mathcal{N}_{i}} a_{i j} c_{i j}(t) a_{i k} c_{i k}(t) \tilde{x}_{i}^{T}(t) \tilde{x}_{k}(t)
\end{aligned}
$$

$$
\begin{aligned}
& +\gamma \sum_{i=1}^{N} \sum_{j \in \mathcal{N}_{i} p \in \mathcal{N}_{j}} a_{i j} c_{i j}(t) a_{j p} c_{j p}(t) \tilde{x}_{i}^{T}(t) \tilde{x}_{j}(t) \\
& -\gamma \sum_{i=1}^{N} \sum_{j \in \mathcal{N}_{i}} \sum_{p \in \mathcal{N}_{j}} a_{i j} c_{i j}(t) a_{j p} c_{j p}(t) \tilde{x}_{i}^{T}(t) \tilde{x}_{p}(t)
\end{aligned}
$$$$
=\sum_{i=1}^{N}\left[\rho-\sum_{j \in \mathcal{N}_{i}} a_{i j} c_{i j}(t)\right.
$$$$
\left.-\gamma \sum_{j \in \mathcal{N}_{i}} \sum_{k \in \mathcal{N}_{i}} a_{i j} c_{i j}(t) a_{i k} c_{i k}(t)\right] \tilde{x}_{i}^{T}(t) \tilde{x}_{i}(t)
$$$$
+\sum_{i=1}^{N} \sum_{j \in \mathscr{N}_{i}}\left[a_{i j} c_{i j}(t)\right.
$$$$
\left.+\gamma \sum_{p \in \mathcal{N}_{j}} a_{i j} c_{i j}(t) a_{j p} c_{j p}(t)\right] \tilde{x}_{i}^{T}(t) \tilde{x}_{j}(t)
$$$$
+\gamma \sum_{i=1}^{N} \sum_{j \in \mathcal{N}_{i}} \sum_{k \in \mathcal{N}_{i}} a_{i j} c_{i j}(t) a_{i k} c_{i k}(t) \tilde{x}_{i}^{T}(t) \tilde{x}_{k}(t)
$$$$
-\gamma \sum_{i=1}^{N} \sum_{j \in \mathcal{N}_{i}} \sum_{p \in \mathcal{N}_{j}} a_{i j} c_{i j}(t) a_{j p} c_{j p}(t) \tilde{x}_{i}^{T}(t) \tilde{x}_{p}(t)
$$$$
\leq \sum_{i=1}^{N}\left[\rho-\sum_{j \in \mathcal{N}_{i}} a_{i j} c_{i j}(t)-\gamma \sum_{j \in \mathscr{N}_{i}} \sum_{k \in \mathcal{N}_{i}} a_{i j} c_{i j}(t) a_{i k} c_{i k}(t)\right]
$$$$
\times \tilde{x}_{i}^{T}(t) \tilde{x}_{i}(t)
$$$$
+\frac{1}{2} \sum_{i=1}^{N} \sum_{j \in \mathscr{N}_{i}}\left[a_{i j} c_{i j}(t)+\gamma \sum_{p \in \mathcal{N}_{j}} a_{i j} c_{i j}(t) a_{j p} c_{j p}(t)\right]
$$$$
\times \tilde{x}_{i}^{T}(t) \tilde{x}_{i}(t)
$$$$
+\frac{1}{2} \sum_{i=1}^{N} \sum_{j \in \mathcal{N}_{i}}\left[a_{i j} c_{i j}(t)+\gamma \sum_{p \in N_{j}} a_{i j} c_{i j}(t) a_{j p} c_{j p}(t)\right]
$$$$
\times \tilde{x}_{j}^{T}(t) \tilde{x}_{j}(t)
$$$$
+\frac{1}{2} \gamma \sum_{i=1}^{N} \sum_{j \in \mathscr{N}_{i}} \sum_{k \in \mathcal{N}_{i}} a_{i j} c_{i j}(t) a_{i k} c_{i k}(t) \tilde{x}_{i}^{T}(t) \tilde{x}_{i}(t)
$$$$
+\frac{1}{2} \gamma \sum_{i=1}^{N} \sum_{j \in \mathscr{N}_{i}} \sum_{k \in \mathscr{N}_{i}} a_{i j} c_{i j}(t) a_{i k} c_{i k}(t) \tilde{x}_{k}^{T}(t) \tilde{x}_{k}(t)
$$$$
+\frac{1}{2} \gamma \sum_{i=1}^{N} \sum_{j \in \mathscr{N}_{i}} \sum_{p \in \mathcal{N}_{j}} a_{i j} c_{i j}(t) a_{j p} c_{j p}(t) \tilde{x}_{i}^{T}(t) \tilde{x}_{i}(t)
$$$$
+\frac{1}{2} \gamma \sum_{i=1}^{N} \sum_{j \in \mathscr{N}_{i}} \sum_{p \in \mathcal{N}_{j}} a_{i j} c_{i j}(t) a_{j p} c_{j p}(t) \tilde{x}_{p}^{T}(t) \tilde{x}_{p}(t)
$$ 


$$
\begin{aligned}
& =\sum_{i=1}^{N}\left[\rho-\sum_{j \in \mathcal{N}_{i}} a_{i j} c_{i j}(t)-\gamma \sum_{j \in \mathcal{N}_{i}} \sum_{k \in \mathcal{N}_{i}} a_{i j} c_{i j}(t) a_{i k} c_{i k}(t)\right] \\
& +\frac{1}{2} \gamma \sum_{j \in \mathcal{N}_{i}} \sum_{p \in \mathcal{N}_{j}} a_{i j} c_{i j}(t) a_{j p} c_{j p}(t) \\
& \times \tilde{x}_{i}^{T}(t) \tilde{x}_{i}(t) \\
& +\sum_{i=1}^{N} \sum_{j \in \mathcal{N}_{i}}\left[a_{i j} c_{i j}(t)+\gamma \sum_{p \in \mathcal{N}_{j}} a_{i j} c_{i j}(t) a_{j p} c_{j p}(t)\right] \\
& +\frac{1}{2} \gamma \sum_{j \in \mathscr{N}_{i}} \sum_{k \in \mathcal{N}_{i}} a_{i j} c_{i j}(t) a_{i k} c_{i k}(t) \\
& \left.+\frac{1}{2} \gamma \sum_{j \in \mathcal{N}_{i}} \sum_{p \in \mathcal{N}_{j}} a_{i j} c_{i j}(t) a_{j p} c_{j p}(t)\right] \\
& \times \tilde{x}_{i}^{T}(t) \tilde{x}_{j}(t) \\
& +\gamma \sum_{i=1}^{N} \sum_{j \in \mathcal{N}_{i}} \sum_{k \in \mathcal{N}_{i}} a_{i j} c_{i j}(t) a_{i k} c_{i k}(t) \tilde{x}_{i}^{T}(t) \tilde{x}_{k}(t) \\
& -\gamma \sum_{i=1}^{N} \sum_{j \in \mathcal{N}_{i}} \sum_{p \in \mathcal{N}_{j}} a_{i j} c_{i j}(t) a_{j p} c_{j p}(t) \tilde{x}_{i}^{T}(t) \tilde{x}_{p}(t) \\
& \leq \sum_{i=1}^{N}\left[\rho-\sum_{j \in \mathcal{N}_{i}} a_{i j} c_{i j}(t)-\gamma \sum_{j \in \mathcal{N}_{i}} \sum_{k \in \mathcal{N}_{i}} a_{i j} c_{i j}(t) a_{i k} c_{i k}(t)\right] \\
& \times \tilde{x}_{i}^{T}(t) \tilde{x}_{i}(t) \\
& +\frac{1}{2} \sum_{i=1}^{N} \sum_{j \in \mathcal{N}_{i}}\left[a_{i j} c_{i j}(t)+\gamma \sum_{p \in \mathcal{N}_{j}} a_{i j} c_{i j}(t) a_{j p} c_{j p}(t)\right] \\
& \times \tilde{x}_{i}^{T}(t) \tilde{x}_{i}(t) \\
& +\frac{1}{2} \sum_{i=1}^{N} \sum_{j \in \mathcal{N}_{i}}\left[a_{i j} c_{i j}(t)+\gamma \sum_{p \in \mathcal{N}_{j}} a_{i j} c_{i j}(t) a_{j p} c_{j p}(t)\right] \\
& \times \tilde{x}_{j}^{T}(t) \tilde{x}_{j}(t) \\
& +\frac{1}{2} \gamma \sum_{i=1}^{N} \sum_{j \in \mathcal{N}_{i}} \sum_{k \in \mathcal{N}_{i}} a_{i j} c_{i j}(t) a_{i k} c_{i k}(t) \tilde{x}_{i}^{T}(t) \tilde{x}_{i}(t) \\
& +\frac{1}{2} \gamma \sum_{i=1}^{N} \sum_{j \in \mathcal{N}_{i}} \sum_{k \in \mathcal{N}_{i}} a_{i j} c_{i j}(t) a_{i k} c_{i k}(t) \tilde{x}_{k}^{T}(t) \tilde{x}_{k}(t) \\
& +\frac{1}{2} \gamma \sum_{i=1}^{N} \sum_{j \in \mathcal{N}_{i}} \sum_{p \in \mathcal{N}_{j}} a_{i j} c_{i j}(t) a_{j p} c_{j p}(t) \tilde{x}_{i}^{T}(t) \tilde{x}_{i}(t) \\
& +\frac{1}{2} \gamma \sum_{i=1}^{N} \sum_{j \in \mathcal{N}_{i}} \sum_{p \in \mathcal{N}_{j}} a_{i j} c_{i j}(t) a_{j p} c_{j p}(t) \tilde{x}_{p}^{T}(t) \tilde{x}_{p}(t) \\
& =\sum_{i=1}^{N}\left[\rho-\sum_{j \in \mathscr{N}_{i}} a_{i j} c_{i j}(t)-\gamma \sum_{j \in \mathscr{N}_{i}} \sum_{k \in \mathcal{N}_{i}} a_{i j} c_{i j}(t) a_{i k} c_{i k}(t)\right. \\
& \times \tilde{x}_{i}^{T}(t) \tilde{x}_{i}(t) \\
& +\frac{1}{2} \sum_{i=1}^{N} \sum_{j \in \mathcal{N}_{i}}\left[a_{i j} c_{i j}(t)+\gamma \sum_{p \in \mathcal{N}_{j}} a_{i j} c_{i j}(t) a_{j p} c_{j p}(t)\right] \\
& \times \tilde{x}_{j}^{T}(t) \tilde{x}_{j}(t) \\
& +\frac{1}{2} \gamma \sum_{i=1}^{N} \sum_{j \in \mathcal{N}_{i}} \sum_{k \in \mathcal{N}_{i}} a_{i j} c_{i j}(t) a_{i k} c_{i k}(t) \tilde{x}_{k}^{T}(t) \tilde{x}_{k}(t) \\
& +\frac{1}{2} \gamma \sum_{i=1}^{N} \sum_{j \in \mathcal{N}_{i}} \sum_{p \in \mathcal{N}_{j}} a_{i j} c_{i j}(t) a_{j p} c_{j p}(t) \tilde{x}_{p}^{T}(t) \tilde{x}_{p}(t) \\
& =\sum_{i=1}^{N}\left[\rho-\frac{1}{2} \sum_{j \in \mathcal{N}_{i}} a_{i j} c_{i j}(t)\right. \\
& -\frac{1}{2} \gamma \sum_{j \in \mathscr{N}_{i}} \sum_{k \in \mathcal{N}_{i}} a_{i j} c_{i j}(t) a_{i k} c_{i k}(t) \\
& \left.+\gamma \sum_{j \in \mathcal{N}_{i}} \sum_{p \in \mathcal{N}_{j}} a_{i j} c_{i j}(t) a_{j p} c_{j p}(t)\right] \\
& \times \tilde{x}_{i}^{T}(t) \tilde{x}_{i}(t) \\
& +\frac{1}{2} \sum_{i=1}^{N} \sum_{j \in \mathcal{N}_{i}}\left[a_{i j} c_{i j}(t)+\gamma \sum_{p \in \mathcal{N}_{j}} a_{i j} c_{i j}(t) a_{j p} c_{j p}(t)\right] \\
& \times \tilde{x}_{j}^{T}(t) \tilde{x}_{j}(t) \\
& +\frac{1}{2} \gamma \sum_{i=1}^{N} \sum_{j \in \mathcal{N}_{i}} \sum_{k \in \mathcal{N}_{i}} a_{i j} c_{i j}(t) a_{i k} c_{i k}(t) \tilde{x}_{k}^{T}(t) \tilde{x}_{k}(t) \\
& +\frac{1}{2} \gamma \sum_{i=1}^{N} \sum_{j \in \mathcal{N}_{i}} \sum_{p \in \mathcal{N}_{j}} a_{i j} c_{i j}(t) a_{j p} c_{j p}(t) \tilde{x}_{p}^{T}(t) \tilde{x}_{p}(t) .
\end{aligned}
$$$$
+\frac{1}{2} \sum_{j \in \mathcal{N}_{i}} a_{i j} c_{i j}(t)
$$

Therefore, 
$\dot{V}_{1}(t)$

$$
\begin{aligned}
& \leq\left[\begin{array}{lll}
\tilde{x}_{1}^{T}(t) \cdots & \tilde{x}_{N}^{T}(t)
\end{array}\right]\left[\begin{array}{cccc}
\rho-\frac{1}{2} l_{11}-\frac{1}{2} \gamma l_{11}^{2}+\gamma \sum_{j=1}^{N} a_{1 j} c_{1 j}(t) l_{j j} & \cdots & 0 & \\
\vdots & \ddots & \vdots & \\
0 & & \cdots & \rho-\frac{1}{2} l_{N N}-\frac{1}{2} \gamma l_{N N}^{2}+\gamma \sum_{j=1}^{N} a_{N j} c_{N j}(t) l_{j j}
\end{array}\right]\left[\begin{array}{c}
\tilde{x}_{1}(t) \\
\widetilde{x}_{2}(t) \\
\vdots \\
\tilde{x}_{N}(t)
\end{array}\right] \\
& +\left[\begin{array}{lll}
\widetilde{x}_{1}^{T}(t) & \cdots & \widetilde{x}_{N}^{T}(t)
\end{array}\right]\left[\begin{array}{cccc}
\frac{1}{2} l_{11}+\frac{1}{2} \gamma l_{11}^{2} & 0 & \cdots & 0 \\
0 & \frac{1}{2} l_{22}+\frac{1}{2} \gamma l_{22}^{2} & \cdots & 0 \\
\vdots & \vdots & \ddots & \vdots \\
0 & 0 & \cdots & \frac{1}{2} l_{N N}+\frac{1}{2} \gamma l_{N N}^{2}
\end{array}\right]\left[\begin{array}{c}
\widetilde{x}_{1}(t) \\
\widetilde{x}_{2}(t) \\
\vdots \\
\widetilde{x}_{N}(t)
\end{array}\right] \\
& +\left[\widetilde{x}_{1}^{T}(t) \cdots \widetilde{x}_{N}^{T}(t)\right]\left[\begin{array}{ccccc}
\frac{1}{2} \gamma \sum_{i=1}^{N} a_{i 1} c_{i 1}(t) l_{i i} & 0 & \cdots & 0 \\
0 & \frac{1}{2} \gamma \sum_{i=1}^{N} a_{i 2} c_{i 2}(t) l_{i i} & \cdots & 0 \\
\vdots & \vdots & \ddots & \vdots \\
0 & 0 & \cdots & \frac{1}{2} \gamma \sum_{i=1}^{N} a_{i N} c_{i N}(t) l_{i i}
\end{array}\right]\left[\begin{array}{c}
\widetilde{x}_{1}(t) \\
\widetilde{x}_{2}(t) \\
\vdots \\
\widetilde{x}_{N}(t)
\end{array}\right] \\
& +\left[\tilde{x}_{1}^{T}(t) \cdots \tilde{x}_{N}^{T}(t)\right]\left[\begin{array}{ccccc}
\frac{1}{2} \gamma \sum_{i=1}^{N} a_{i 1} c_{i 1}(t) l_{i i} & 0 & \cdots & 0 \\
0 & \frac{1}{2} \gamma \sum_{i=1}^{N} a_{i 2} c_{i 2}(t) l_{i i} & \cdots & 0 \\
\vdots & \vdots & \ddots & \vdots \\
0 & 0 & \cdots & \frac{1}{2} \gamma \sum_{i=1}^{N} a_{i N} c_{i N}(t) l_{i i}
\end{array}\right]\left[\begin{array}{c}
\tilde{x}_{1}(t) \\
\tilde{x}_{2}(t) \\
\vdots \\
\tilde{x}_{N}(t)
\end{array}\right] \\
& =\left[\begin{array}{lll}
\widetilde{x}_{1}^{T}(t) & \cdots & \widetilde{x}_{N}^{T}(t)
\end{array}\right]\left[\begin{array}{ccc}
\rho+\gamma\left(W_{1}+V_{1}\right) & \cdots & 0 \\
\vdots & \ddots & \vdots \\
0 & \cdots & \rho+\gamma\left(W_{N}+V_{N}\right)
\end{array}\right]\left[\begin{array}{c}
\tilde{x}_{1}(t) \\
\tilde{x}_{2}(t) \\
\vdots \\
\tilde{x}_{N}(t)
\end{array}\right],
\end{aligned}
$$

where $l_{i i}=-u_{i i}=\sum_{j=1, j \neq i}^{N} a_{i j} c_{i j}(t), W_{i}=\sum_{j=1}^{N} a_{i j} c_{i j}(t) l_{j j}, V_{j}=$ $\sum_{i=1}^{N} a_{i j} c_{i j}(t) l_{i i}, i, j=1,2, \ldots, N$.

Differentiating $V_{2}(t)$, we get

$$
\begin{aligned}
\dot{V}_{2}(t) & =(1-m) c_{i j}(t) \dot{c}_{i j}(t) \\
& =\sum_{i=1}^{N} \sum_{j \in \mathcal{N}_{i}}(1-m) c_{i j}(t)
\end{aligned}
$$

$$
\times\left\{\sum_{p \in \mathcal{N}_{j}} a_{i j} a_{j p} c_{j p}(t)\left[x_{i}(t)-\bar{x}(t)\right]^{T}\right.
$$$$
\times\left[x_{i}(t)-\bar{x}(t)\right]
$$$$
+\sum_{k \in \mathcal{N}_{i}} a_{i j} a_{i k} c_{i k}(t)\left[x_{k}(t)-\bar{x}(t)\right]^{T}
$$ 


$$
\begin{gathered}
\left.\times\left[x_{k}(t)-\bar{x}(t)\right]\right\} \\
=(1-m) \sum_{i=1}^{N} \sum_{j \in \mathcal{N}_{i}} \sum_{p \in \mathcal{N}_{j}} a_{i j} c_{i j}(t) a_{j p} c_{j p}(t) \tilde{x}_{i}^{T}(t) \tilde{x}_{i}(t) \\
+(1-m) \sum_{i=1}^{N} \sum_{j \in \mathcal{N}_{i}} \sum_{k \in \mathcal{N}_{i}} a_{i j} c_{i j}(t) a_{i k} c_{i k}(t) \tilde{x}_{k}^{T}(t) \tilde{x}_{k}(t) \\
=\left[\begin{array}{lll}
\tilde{x}_{1}^{T}(t) & \cdots & \left.\tilde{x}_{N}^{T}(t)\right]
\end{array}\right.
\end{gathered}
$$

$$
\times\left[\begin{array}{ccc}
(1-m)\left(W_{1}+V_{1}\right) & \cdots & 0 \\
\vdots & \ddots & \vdots \\
0 & \cdots & (1-m)\left(W_{N}+V_{N}\right)
\end{array}\right]
$$$$
\times\left[\begin{array}{c}
\tilde{x}_{1}(t) \\
\tilde{x}_{2}(t) \\
\vdots \\
\tilde{x}_{N}(t)
\end{array}\right]
$$

$$
\dot{V}(t)=\dot{V}_{1}(t)+\dot{V}_{2}(t)
$$$$
\leq\left[\begin{array}{lll}
\tilde{x}_{1}^{T}(t) & \cdots & \tilde{x}_{N}^{T}(t)
\end{array}\right] \times\left[\begin{array}{ccc}
\rho+(\gamma+1-m)\left(W_{1}+V_{1}\right) & \cdots & 0 \\
\vdots & \ddots & \vdots \\
0 & \cdots & \rho+(\gamma+1-m)\left(W_{N}+V_{N}\right)
\end{array}\right]
$$$$
\times\left[\begin{array}{c}
\tilde{x}_{1}(t) \\
\tilde{x}_{2}(t) \\
\vdots \\
\tilde{x}_{N}(t)
\end{array}\right]<0
$$

Combining $\dot{V}_{1}(t)$ and $\dot{V}_{2}(t)$, and since $m>0$ is sufficiently large, then we can have where $l_{i i}, W_{i}, V_{j}$ are defined as before.

So,

$$
\lim _{t \rightarrow \infty}\left\|x_{i}(t)-\bar{x}(t)\right\|=0
$$

These complete the proof.

Theorem 9. Network (4) solves an agreement problem faster than the same network without the state predictor.

Proof. The proof is similar to that of Theorem 2 in [33].

\section{Simulations}

In this section, we give the numerical simulations to illustrate the analytical results.

Consider a small network with the undirected topology described as the following symmetric matrix:

$$
A=\left[\begin{array}{cccc}
0 & 0.0964 & 0.0757 & 0.0570 \\
0.0964 & 0 & 0.1199 & 0.1396 \\
0.0757 & 0.1199 & 0 & 0.0581 \\
0.0570 & 0.1396 & 0.0581 & 0
\end{array}\right]
$$

where all nodes of the pagebreak network and their synchronous goal will obey the same nonlinear dynamics described as the Lorenz system:

$$
f(x(t))=f\left(x^{1}, x^{2}, x^{3}\right)=\left\{\begin{array}{l}
\dot{x}^{1}=10\left(x^{2}-x^{1}\right) \\
\dot{x}^{2}=28 x^{1}-x^{1} x^{3}-x^{2} \\
\dot{x}^{3}=x^{1} x^{2}-\frac{8}{3} x^{3}
\end{array}\right.
$$

as shown in Figure 1.

Figure 2 describes the convergence of the state errors on the $x$-axis, $y$-axis, and $z$-axis, respectively. From this figure, we can see that all nodes of the above network can synchronize to the synchronous state gradually. With the same initial state and the same nonlinear dynamics, influenced by the same adaptive strategy, it can be easy to find as in Figure 3 that the network with a state predictor can also synchronize to the synchronous state and be faster than the network without a state predictor.

\section{Conclusion}

In this paper, we have investigated the adaptive synchronization of complex dynamical networks with nonlinear dynamics. By introducing local decentralized adaptive strategies to the coupling strengths, we have proved that the network with a state predictor can synchronize to the synchronous state. 


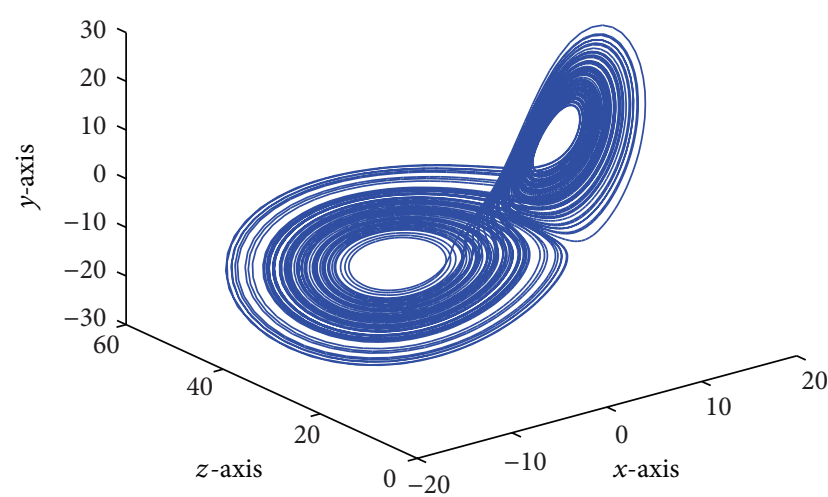

FIGURE 1: The nonlinear dynamics of the network.
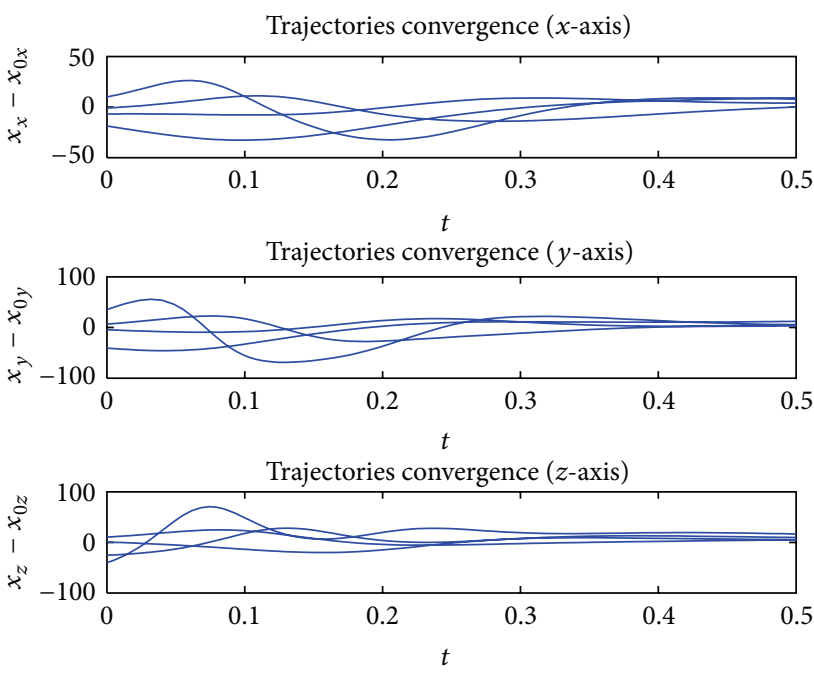

Figure 2: The trajectories of four agents in the dynamical network without a state predictor.

Trajectories convergence ( $x$-axis)
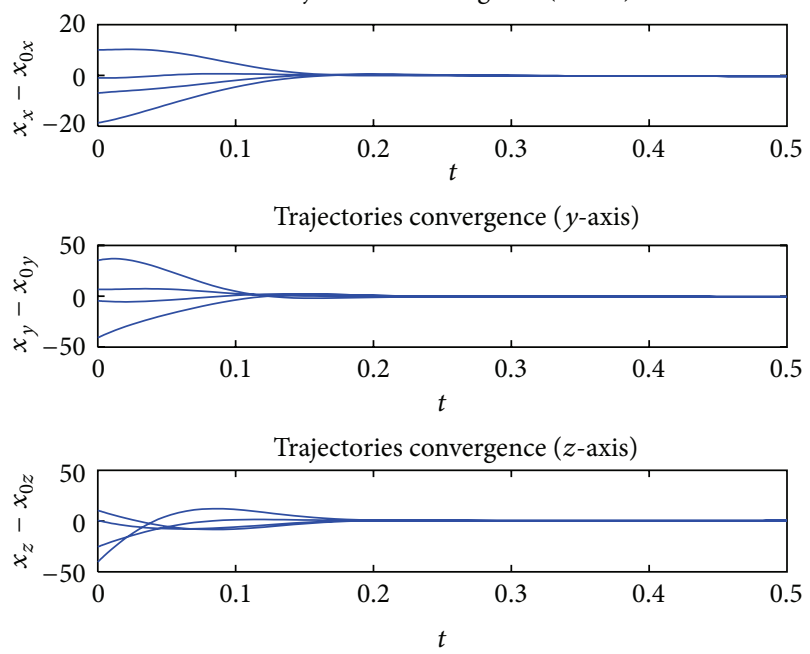

FIGURE 3: The trajectories of four agents in the dynamical network with a state predictor when $\gamma=200$.

\section{Acknowledgments}

This work was supported in part by the National Natural Science Foundation of China under Grant no. 61304049, no. 61174116, the Beijing Natural Science Foundation Program 4132021, Science and Technology Development Plan Project of Beijing Education Commission (no. KM201310009011), and Funding Project for Academic Human Resources Development in Institutions of Higher Learning Under the Jurisdiction of Beijing Municipality (PHR201108055).

\section{References}

[1] X. F. Wang and G. Chen, "Pinning control of scale-free dynamical networks," Physica A, vol. 310, no. 3-4, pp. 521-531, 2002.

[2] P. De Lellis, M. di Bernardo, and F. Garofalo, "Synchronization of complex networks through local adaptive coupling," Chaos, vol. 18, no. 3, Article ID 037110, p. 8, 2008.

[3] H. Su, Z. Rong, M. Chen, X. Wang, G. Chen, and H. Wang, "Decentralized adaptive pinning control for cluster synchronization of complex dynamical networks," EEE Transactions on Cybernetics, vol. 43, pp. 394-399, 2013.

[4] W. Yu, G. Chen, J. Lü, and J. Kurths, "Synchronization via pinning control on general complex networks," SIAM Journal on Control and Optimization, vol. 51, no. 2, pp. 1395-1416, 2013.

[5] B. Liu, X. Wang, H. Su, Y. Gao, and L. Wang, "Adaptive secondorder consensus of multi-agent systems with heterogeneous nonlinear dynamics and timevarying delays," Neurocomputing, vol. 118, pp. 289-300, 2013.

[6] H. Su and W. Zhang, "Second-order consensus of multiple agents with coupling delay," Communications in Theoretical Physics, vol. 51, pp. 101-109, 2009.

[7] B. Liu, W. Hu, J. Zhang, and H. Su, "Controllability of discretetime multi-agent systems with multiple leaders on fixed networks," Communications in Theoretical Physics, vol. 58, pp. 856862, 2012.

[8] H. Su, M. Chen, J. Lam, and Z. Lin, "Semi-global leaderfollowing consensus of linear multi-agent systems with input saturation via low gain feedback," IEEE Transactions on Circuits and Systems, vol. 60, pp. 1881-1889, 2013.

[9] G. Wen, Z. Duan, W. Yu, and G. Chen, "Consensus of multiagent systems with nonlinear dynamics and sampled-data information: a delayed-input approach," International Journal of Robust and Nonlinear Control, vol. 23, no. 6, pp. 602-619, 2013.

[10] W. Yu, P. DeLellis, G. Chen, M. di Bernardo, and J. Kurths, "Distributed adaptive control of synchronization in complex networks," IEEE Transactions on Automatic Control, vol. 57, no. 8, pp. 2153-2158, 2012.

[11] H. Su, M. Chen, X. Wang, H. Wang, and N. V. Valeyev, "Adaptive cluster synchronization of coupled harmonic oscillators with multiple leaders," IET Control Theory and Applications, vol. 7, pp. 765-772, 2013.

[12] M. Wang, H. Su, M. Zhao, M. Chen, and H. Wang, "Flocking of multiple autonomous agents with preserved network connectivity and heterogeneous nonlinear dynamics," Neurocomputing, vol. 115, pp. 169-177, 2013.

[13] W. Zhang, H. Su, and J. Wang, "Computation of upper bounds for the solution of continuous algebraic riccati equations," Circuits, Systems and Signal Processing, vol. 32, pp. 1477-1488, 2013. 
[14] W. Yu, G. Chen, and J. Lü, "On pinning synchronization of complex dynamical networks," Automatica, vol. 45, no. 2, pp. 429-435, 2009.

[15] P. Lin and Y. Jia, "Consensus of second-order discrete-time multi-agent systems with nonuniform time-delays and dynamically changing topologies," Automatica, vol. 45, no. 9, pp. 21542158, 2009.

[16] Z. Tang, T. Huang, J. Shao, and J. Hu, "Consensus of secondorder multi-agent systems with nonuniform time-varying delays," Neurocomputing, vol. 97, pp. 410-414, 2012.

[17] H. Su, X. Wang, and Z. Lin, "Flocking of multi-agents with a virtual leader," IEEE Transactions on Automatic Control, vol. 54, no. 2, pp. 293-307, 2009.

[18] Z. Hou, L. Cheng, and M. Tan, "Decentralized robust adaptive control for the multiagent system consensus problem using neural networks," IEEE Transactions on Systems, Man, and Cybernetics-Patr B, vol. 39, pp. 636-647, 2009.

[19] P. De Lellis, M. di Bernardo, and F. Garofalo, "Synchronization of complex networks through local adaptive coupling," Chaos, vol. 18, no. 3, Article ID 037110, p. 8, 2008.

[20] H. Su, X. Wang, and G. Chen, "Rendezvous of multiple mobile agents with preserved network connectivity," Systems \& Control Letters, vol. 59, no. 5, pp. 313-322, 2010.

[21] H. Su, G. Chen, X. Wang, and Z. Lin, "Adaptive secondorder consensus of networked mobile agents with nonlinear dynamics," Automatica, vol. 47, no. 2, pp. 368-375, 2011.

[22] Y. Hu, H. Su, and J. Lam, "Adaptive consensus with a virtual leader of multiple agents governed by locally Lipschitz nonlinearity," International Journal of Robust and Nonlinear Control, vol. 23, no. 9, pp. 978-990, 2013.

[23] W. Zhang, H. Su, H. Wang, and Z. Han, "Full-order and reduced-order observers for one-sided Lipschitz nonlinear systems using Riccati equations," Communications in Nonlinear Science and Numerical Simulation, vol. 17, no. 12, pp. 4968-4977, 2012.

[24] O. Faydasicok and S. Arik, "A new robust stability cirterion for dynamical neural networks with multiple time delays," Neurocomputing, vol. 99, pp. 290-297, 2013.

[25] H. Su, "Flocking in multi-agent systems with multiple virtual leaders based only on position measurements," Communications in Theoretical Physics, vol. 57, pp. 801-807, 2012.

[26] J. Zhang, M. Li, and B. Liu, "Synchronization of a complex network for neighboring agents with time-varying delays," Communications in Computer and Information Science, vol. 225, pp. 73-81, 2011.

[27] B. Liu, T. Chu, L. Wang, Z. Zuo, G. Chen, and H. Su, "Controllability of switching networks of multi-agent systems," International Journal of Robust and Nonlinear Control, vol. 22, no. 6, pp. 630-644, 2012.

[28] W. Zhang, H. Su, Y. Liang, and Z. Han, "Robust stability test for uncertain discrete-time systems: a descriptor system approach," Latin American Applied Research, vol. 41, pp. 359-364, 2011.

[29] W. Yu, "A LMI-based approach to global asymptotic stability of neural networks with time varying delays," Nonlinear Dynamics, vol. 48, no. 1-2, pp. 165-174, 2007.

[30] H. Su, X. Wang, and Z. Lin, "Synchronization of coupled harmonic oscillators in a dynamic proximity network," Automatica, vol. 45, no. 10, pp. 2286-2291, 2009.

[31] R. Olfati-Saber and R. M. Murray, "Consensus problems in networks of agents with switching topology and time-delays," IEEE Transactions on Automatic Control, vol. 49, no. 9, pp. 15201533, 2004.
[32] H. Su, X. Wang, and G. Chen, "A connectivity-preserving flocking algorithm for multi-agent systems based only on position measurements," International Journal of Control, vol. 82, no. 7, pp. 1334-1343, 2009.

[33] G. Xi, W. Huang, and X. Li, "Consensus of multi-agent system with state predictor," Control and Decision, vol. 25, pp. 669-772, 2010.

[34] K. Peng, H. Su, and Y. Yang, "Coordinated control of multiagent systems with a varying-velocity leader and input saturation," Communications in Theoretical Physics, vol. 52, pp. 449456, 2009.

[35] H. Su, X. Wang, and W. Yang, "Flocking in multi-agent systems with multiple virtual leaders," Asian Journal of Control, vol. 10, no. 2, pp. 238-245, 2008.

[36] H. Su, N. Zhang, M. Z. Q. Chen, H. Wang, and X. Wang, "Adaptive flocking with a virtual leader of multiple agents governed by locally Lipschitz nonlinearity," Nonlinear Analysis: Real World Applications, vol. 14, no. 1, pp. 798-806, 2013.

[37] B. Liu, T. Chu, and L. Wang, "Stability and oscillation of swarm with interaction time delays," in Proceedings of the American Control Conference, pp. 4600-4605, 2007.

[38] C. Godsil and G. Royle, Algebraic Graph Theory, vol. 207, Springer, New York, NY, USA, 2001. 


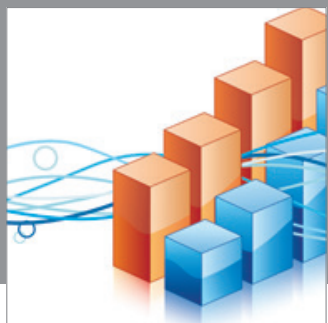

Advances in

Operations Research

mansans

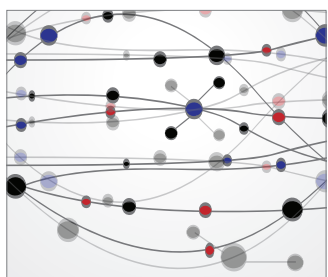

The Scientific World Journal
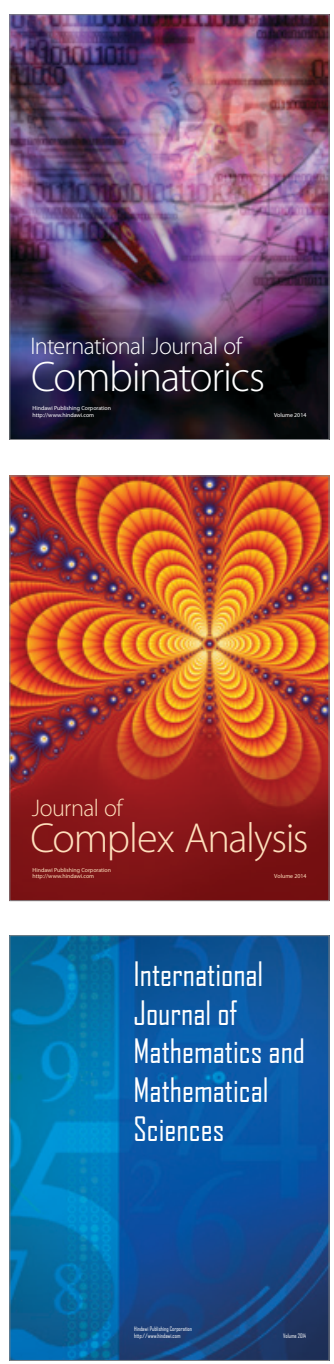
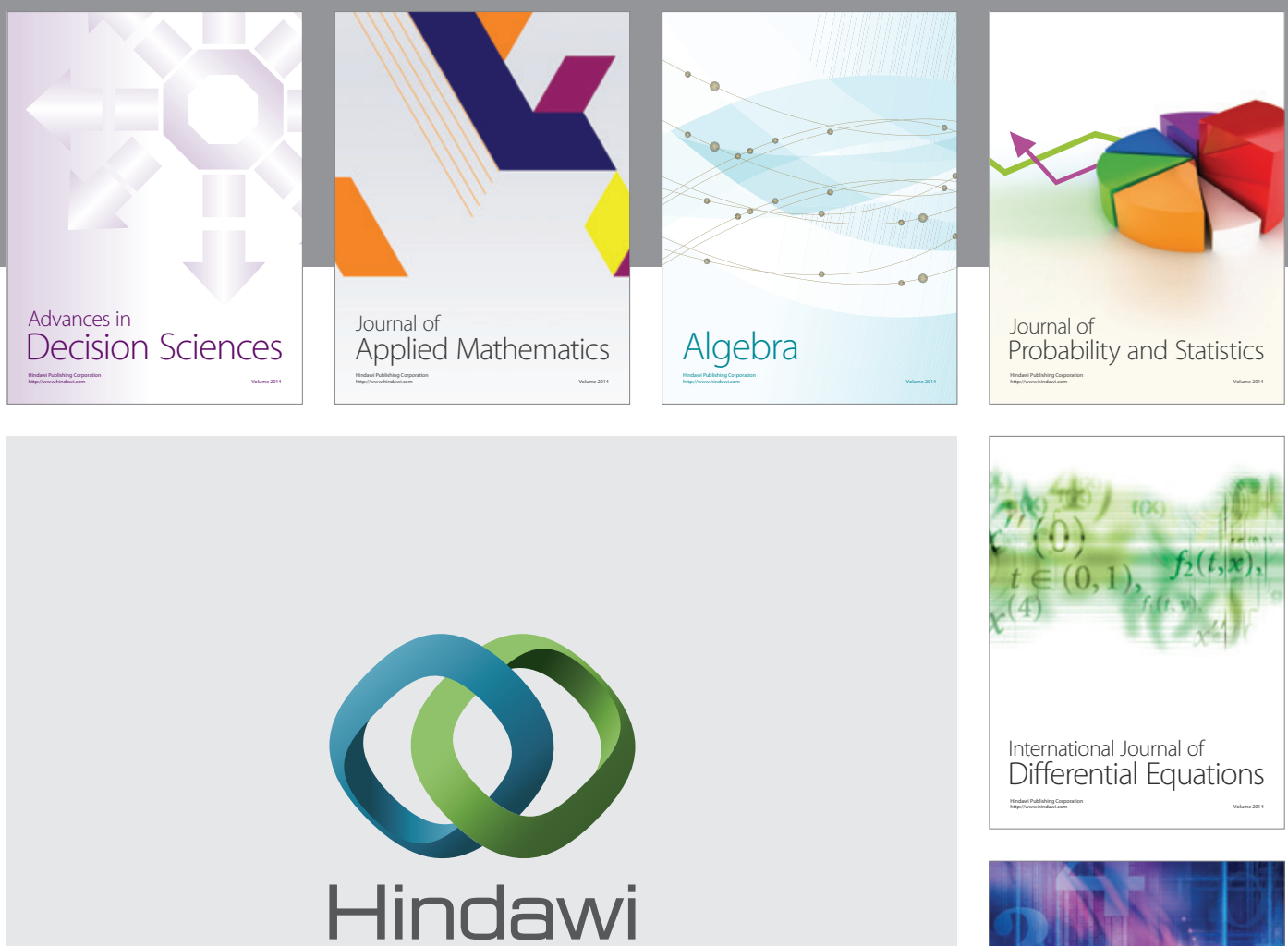

Submit your manuscripts at http://www.hindawi.com
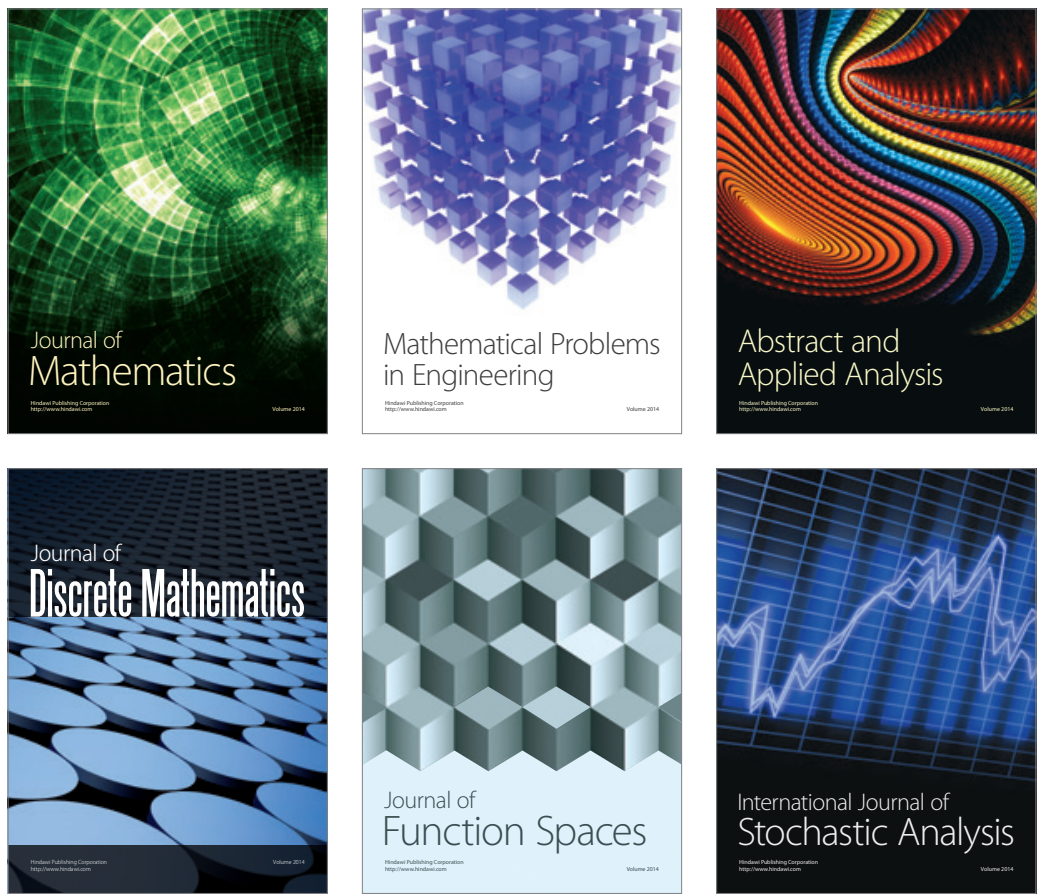

Journal of

Function Spaces

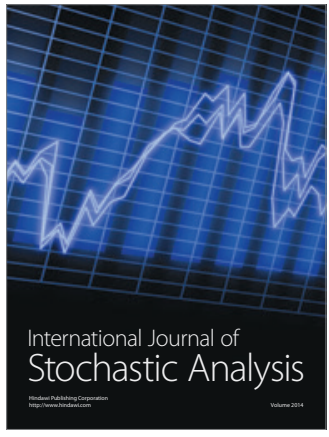

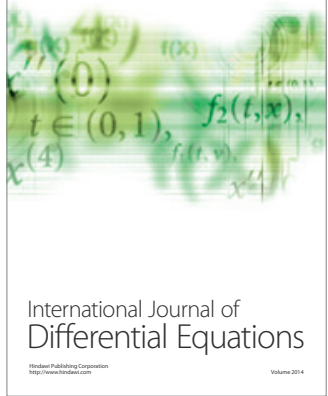
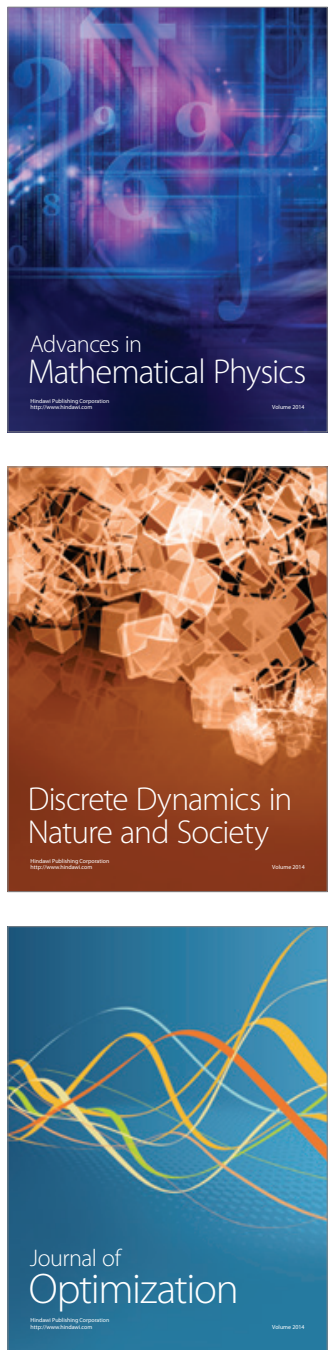